\title{
Pesticide Mineralization in Water Using Silver Nanoparticles Incorporated on Polyurethane Foam
}

\author{
G. Manimegalai ${ }^{1}$, S. Shanthakumar ${ }^{2}$, Chandan Sharma ${ }^{3}$ \\ ${ }^{1}$ Environmental Engineering Division, School of Mechanical and Building Sciences, \\ VIT University, Vellore-632014, India \\ maniganesh87@gmail.com \\ ${ }^{2}$ Environmental Engineering Division, School of Mechanical and Building Sciences, \\ VIT University, Vellore-632014, India \\ sskumariit@gmail.com \\ ${ }^{3}$ Chemical Engineering Division, School of Mechanical and Building Sciences, \\ VIT University, Vellore-632014, India \\ chandan1816@gmail.com
}

\begin{abstract}
In present day scenario, pesticides are widely used for pest control in agriculture, because of which, the drinking water sources (both surface and ground water) gets contaminated. Majority of the pesticides are defiant to biodegradation and are found to be carcinogenic in nature even at trace levels. Various conventional methods employed for removal of pesticide are surface adsorption, photo-catalysis, membrane separation and biodegradation. However, these methods have disadvantages such as time consumption and expensive. Application of nanoparticles alleviates both of the above mentioned drawbacks. The nanoparticles need to be incorporated on a support to prevent the presence of it in the purified water after mineralization of pesticides. Previous researchers employed activated carbon and alumina as support for silver nanoparticles in pesticide mineralization. However, not many studies have been carried out on polyurethane foam (PUF) as support for silver nanoparticles in the mineralization of pesticides (Chlorpyrifos \& Malathion) in water. Hence, a detailed study has been carried out to estimate the mineralization potential of silver nanoparticles supported on PUF. Silver nanoparticles synthesized using trisodium citrate as reducing agent was used for incorporating into polyurethane foam. Polyurethane foam was found to be having saturation for supporting AgNPs. The pesticide concentration found to be directly proportional to mineralization time.
\end{abstract}

Keywords: Pesticide, Nanoparticles, Polyurethane foam, Mineralization

\section{Introduction}

Water pollution due to pesticides is a critical problem in developing countries since they are deliberately used to control pest in agriculture and public health and hold a unique position among contaminants found in water[1]. Runoff from agricultural fields, industrial wastes and orchards treated with pesticides are the various sources of pesticides in water[2]. Pesticides comprise of different classes such as insecticides, fungicides, herbicides, rodenticides etc. and due to their widespread use, they leach into surface and ground water and hence, present in drinking water as well. They persist in the environment and pose a significant health threat. Among the possible effects related to this exposure, genetic damage has important health implications for the induction of lung cancer, non-Hodgkin's lymphoma, pancreatic cancer, bladder cancer and leukaemia[3-4].

The awareness about the risks associated with drinking water contamination is increasing in the present scenario due to which the allowable limits are being revised and the permissible limits are expected to reach molecular levels in the coming years. In spite of the negative perception of the public, pesticides are still going to be utilised for many decades to ensure the food supply for the ever growing world population[5]. Hence, it becomes essential to develop new technologies which are capable of removing pesticides even at ppm or ppb levels.

Previously, the researchers employed various methods for pesticide removal which includes photo-catalysis, biodegradation, adsorption and membrane separation[2]. These methods are disadvantageous either due to their time consumption or expensiveness. The applications of nanotechnology are increasing in all areas of science and technology, including the field of environmental studies and treatment[6]. Nanoparticles are usually referred to as clusters of atoms in the size range of 1-100 $\mathrm{nm}[7]$. Nanoparticles exhibit a completely new or improved properties compared to larger particles of bulk material and these novel properties are derived due to the variation in specific characteristics such as size, ionic state, distribution and morphology of the particles. Nanoparticles exhibit a higher surface area-to-volume ratio with decrease in the size of particles[8]. It has been well established that the metallic nanoparticles such as zero-valent iron, copper, silver and gold have unique catalytic activity in the mineralization of halocarbons and other organic as well as inorganic contaminants.

Metal nanoparticles can be prepared by two routes such as physical and chemical approach. Physical methods are evaporation/condensation and laser ablation etc. chemical methods can be subdivided into three types such as classical chemicals using the well-known chemical reducing 
substances (hydrazine, sodium borohydride, hydrogen etc.), radiation chemicals where the reduction process is initiated by solvated electrons generated by the ionized radiation and green chemicals using naturally occurring reducing agents such as polysaccharides, plant extract or culture supernatant of bacteria and fungi[7].

However, not many studies have been carried out on polyurethane foam (PUF) as support for silver nanoparticles in the mineralization of pesticides in water. Hence, a detailed study has been carried out to estimate the mineralization potential of silver nanoparticles supported on PUF and the critical evaluation of the results are presented in this paper.

\section{Materials and Methods}

\subsection{Chemicals and Reagents}

Silver nitrate, diethyl amine, trisodium citrate, and hexane were purchased from Thomas baker, India. All the reagents used were of analytical reagent grade and were used as received. Milli Q water was used for the synthesis of silver nanoparticles and double distilled water was used for the mineralization of pesticides. Commercially available pesticides such as Chlorpyrifos and Malathion were obtained from local pesticide shop. Polyurethane foam was purchased from local store.

\subsection{Synthesis of Silver Nanoparticles}

$200 \mathrm{~mL}$ of $5 \mathrm{mM}$ silver nitrate was diluted into 1L in water and was heated until it begins to boil. $40 \mathrm{~mL}$ of $1 \%$ trisodium citrate solution was added and heated continuously until the colour changed to pale yellow. The solution was cooled to room temperature[9]. Aqueous nanoparticles were coated as film on glass slides for atomic force microscope (AFM) analysis.

\subsection{Incorporation of AgNPs onto Polyurethane foam}

Polyurethane foams were soaked in silver nanoparticles solution overnight. For the saturated coating of $20 \mathrm{~cm} \times 25$ $\mathrm{cm}$ foam $\sim 1.5 \mathrm{~L}$ of nanoparticles solution was required[10]. The sheets were washed repeatedly with water to remove any adsorbed ions like citrate and were air dried.

\subsection{Extraction of pesticide using hexane}

After the treatment of pesticide contaminated water with AgNPs, $75 \mathrm{~mL}$ of hexane was added to $500 \mathrm{~mL}$ of the treated water. It was mixed well and kept for 30 minutes for the separation into layers of hexane with pesticide and water. The hexane with the extracted pesticide was kept out for evaporation of hexane till the final volume was up to 3 $4 \mathrm{~mL}$. It was made up to $10 \mathrm{~mL}$ with hexane and used to find out the absorbance value using UV-Visible spectrophotometer[11].

\subsection{Mineralization of Pesticide using Polyurethane Foam (PUF) incorporated with AgNPs}

Various required concentrations (1, 2 and $3 \mathrm{ppm}$ ) of pesticide solutions were prepared. AgNPs incorporated PUF (20 cm x $25 \mathrm{~cm}$ ) was cut into pieces and immersed in the prepared pesticide solution.

\subsection{Pesticide Estimation}

The estimation of Chlorpyrifos was done by measuring the absorbance peak at $292 \mathrm{~nm}$ and for Malathion at 267 $\mathrm{nm}[11]$. $3.5 \mathrm{~mL}$ aliquots of the solution were taken out at equal time intervals (20 minutes) for analysis by UVVisible spectroscopy and the solutions were poured back after measuring the absorbance. When the absorbance was found to be negligible, the supports incorporated with nanoparticles were taken out of the water. To confirm the complete mineralization process, hexane was used to extract even trace amounts of pesticide from the treated water. $75 \mathrm{~mL}$ of hexane was required for each $500 \mathrm{~mL}$ of treated water.

\section{Results and Discussion}

\subsection{Synthesis of Silver Nanoparticles using trisodium citrate as Reducing Agent}

Fig. 1 shows the AFM image of silver nanoparticles. The size of the nanoparticles is in the range of $60-90 \mathrm{~nm}$.

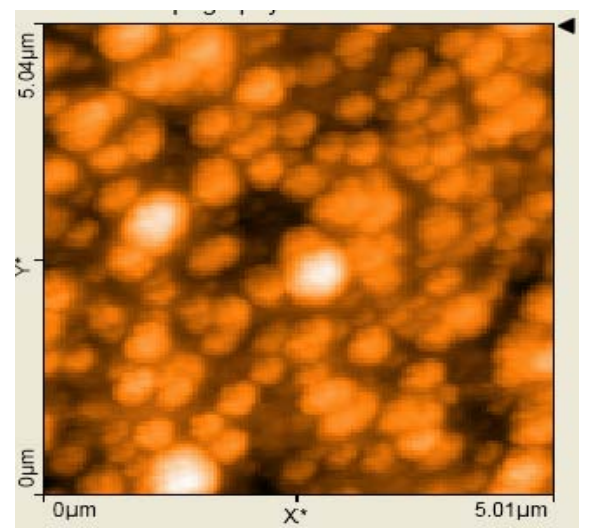

Figure 1. AFM image of AgNPs $\left(5 x 5 \mu m^{2}\right)$

\subsection{Incorporation of Silver Nanoparticles onto Polyurethane Foam}

Fig. 2 depicts the image of Polyurethane foam before and after the incorporation of nanoparticles. Optical microscopic image of polyurethane foam Fig. 3 (before and after incorporation of AgNPs) shows the detailed suface area where nanoparticles get attached.

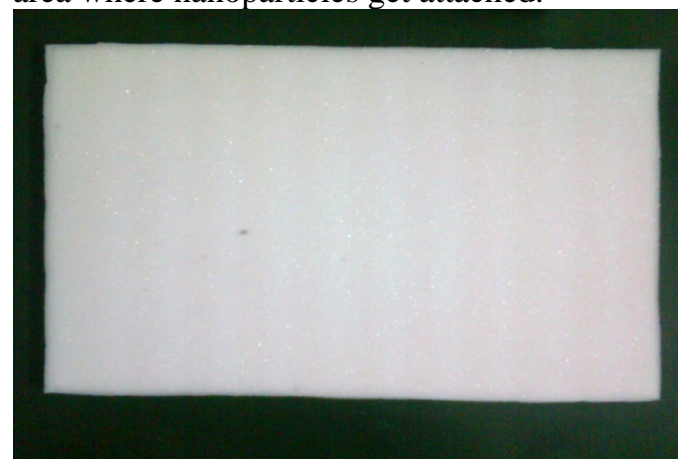

(a) 


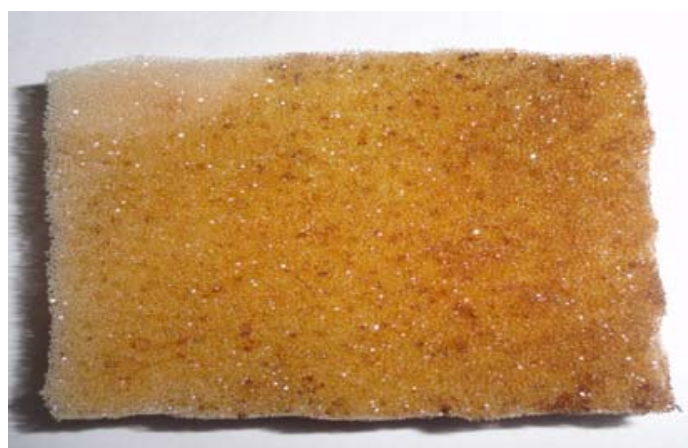

(b)

Figure 2. Polyurethane foam before (a) and after (b) incorporation of AgNPs

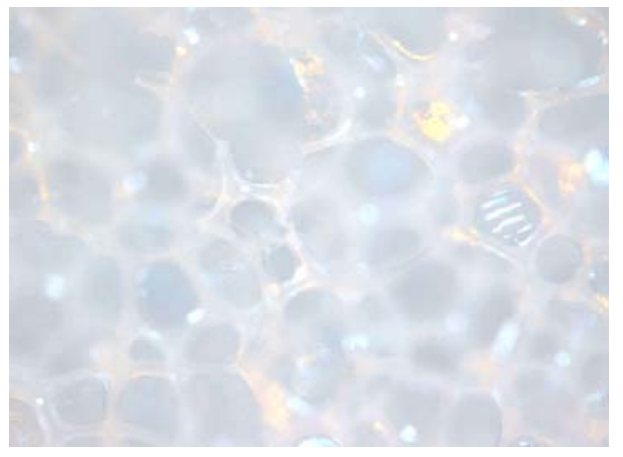

(a)

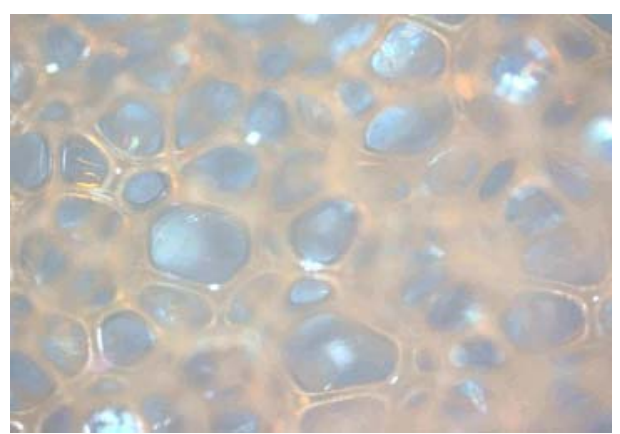

(b)

Figure 3. Optical microscopic image of PUF before (a) and after (b) incorporation of AgNPs

Due to the incorporation of nanoparticles, the white surface of the polyurethane foam became yellow coloured.

\subsection{Mineralization of Chlorpyrifos and Malathion using AgNPs supported on PUF}

It can be noted from Fig. 4 that the complete mineralization time for 1 ppm, 2 ppm and 3 ppm Chlorpyrifos were found to be 100 mins, 140 mins and 180 mins respectively. Similarly, the mineralization time for 1 ppm, 2 ppm and 3 ppm Malathion were found to be 100 mins, 140 mins and 200 mins respectively (Fig.5).

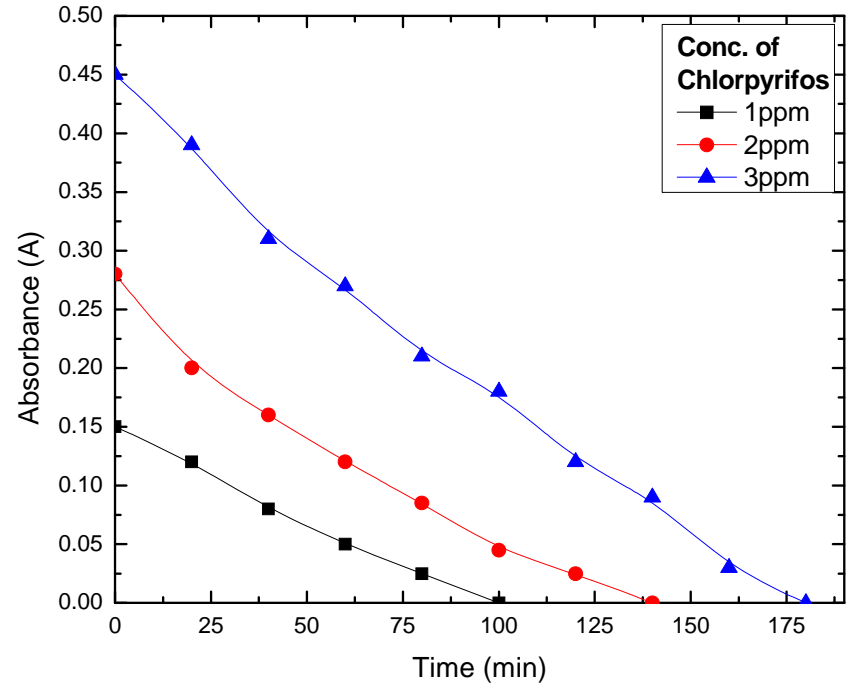

Figure 4. Mineralization of Chlorpyrifos at different concentrations using AgNPs supported on PUF

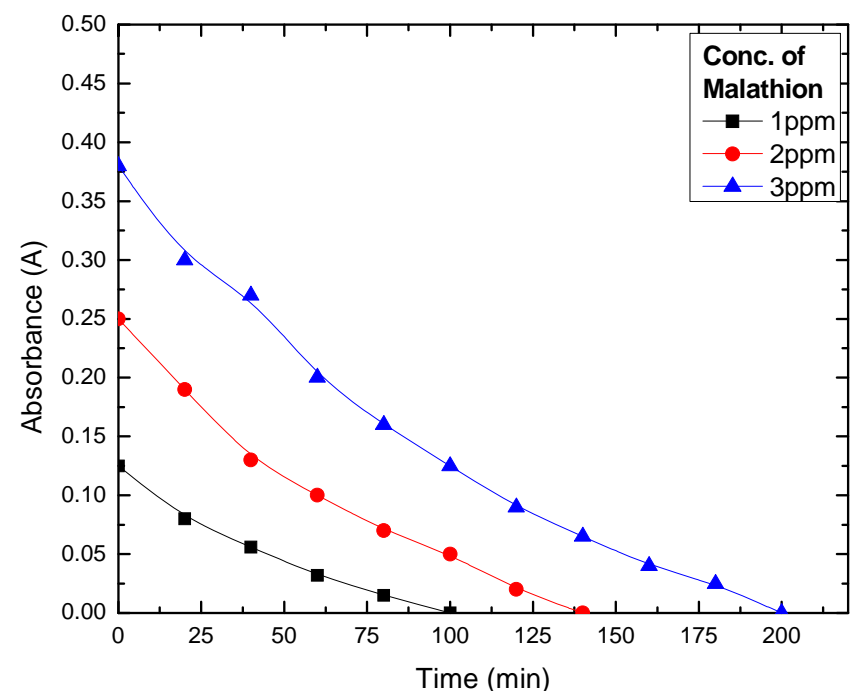

Figure 5. Mineralization of Malathion at different concentrations using AgNPs supported on PUF

\section{Conclusion}

Based on the results and discusssion, it has been demonstrated that pesticide concentration influence the mineralization time and it does not discriminate different pesticides. Since the normal concentration of pesticide contaminated drinking water sources are in the range of $\mathrm{ppb}$ which is far lower than 1-3 ppm, the silver nanoparticles supported on polyurethane foam can be used for the removal of pesticides in the rural areas where the pesticide contamination is prevalent.

\section{References}

[1] A. W. A. Brown, Ecology of Pesticides, John wiley \& sons, New York, 1977. 
[2] N. Savage, M. D. J. Duncan, A. Street and R. Sustich, Nanotechnology Applications for Clean Water, William Andrew Inc, 2009.

[3] N. Itot, A. Hagiwara, S. Tamano, M. Futacuchis, K. Imaidas and T. Shiraij, "Effects of Pesticide Mixtures at the Acceptable Daily Intake Levels on Rat Carcinogenesis," Journal of Food and Chemical Toxicology, 34, pp. 1091-1096, 1996.

[4] N. Sailaja, M. Chandrasekhar, P. V. Rekhadevi, M. Mahbooba, M. F. Rahmana, S. B. Vuyyuri, K. Danadevi, S. A. Hussain and P. Grover, "Genotoxic evaluation of workers employed in pesticide production,” Journal of MutationResearch.,609, pp. 7480, 2006.

[5] C. J. Wang and Z. Q. Liu, "Foliar uptake of pesticidesPresent status and future challenge Pesticide,” Journal of Biochemistry and Physiology, 87, pp. 1-8, 2007.

[6] K. Watlington, Emerging Nanotechnologies for Site Remediation and Wastewater Treatment, USEPA Report, 2005.

[7] E. N. K. M. Abou, A. Eftaiha, A. Al-Warthan and R. A. A. Ammar, "Synthesis and applications of silver nanoparticles,” .Arabian Journal of Chemistry, 3, pp. 135-140, 2009.

[8] S. Gurunathana, K. Kalishwaralala, R. Vaidyanathana, V. Deepaka, S. R. Pandiana, J. Muniyandia, N. Hariharana and S. H. Eomb, "Biosynthesis, purification and characterization of silver nanoparticles using Escherichia coli," Journal of Colloids and Surfaces B: Biointerfaces, 74, pp. 328-335, 2009.

[9] P. V. Kamat, M. Flumiani and G. V. Hartland, "Picosecond dynamics of silver nanoclusters. Light induced fragmentation and photoejection of electrons," Journal of Physical Chemistry, B102, pp. 3123-3128, 1998.

[10] P. Jain and T. Pradeep, "Potential of Silver Nanoparticle-coated Polyurethane Foam as an antibacterial water filter," Biotechnology and Bioengineering, 90(1), pp. 59-63, 2005.

[11] S. Nair and T. Pradeep, "Extraction of Chlorpyrifos and Malathion from Water by Metal Nanoparticles," .Journal of Nanoscience and Nanotechnology, 7, pp.1-7, 2007. 